\title{
AUTOMORPHISMS OF THE PRO JECTIVE UNIMODULAR GROUP
}

BY

L. K. HUA AND I. REINER

Notation. Let $\mathfrak{M}_{n}$ denote the group of $n \times n$ integral matrices of determinant \pm 1 (the unimodular group). By $\mathfrak{M}_{n}^{+}$we denote that subset of $\mathfrak{M}_{n}$ where the determinant is $+1 ; \mathfrak{M}_{n}^{-}$is correspondingly defined. Let $\mathfrak{P}_{2 n}$ be obtained from $\mathfrak{M}_{2 n}$ by identifying $+X$ and $-X, X \in \mathfrak{M}_{2 n}$. (This is the same as considering the factor group of $\mathfrak{M}_{2 n}$ by its centrum.) We correspondingly obtain $\mathfrak{P}_{2 n}^{+}$and $\mathfrak{P}_{2 n}^{-}$from $\mathfrak{M}_{2 n}^{+}$and $\mathfrak{M}_{2 n}^{-}$. Let $I^{(n)}$ (or briefly $I$ ) be the identity matrix in $\mathfrak{M}_{n}$, and let $X^{\prime}$ denote the transpose of $X$. The direct sum of $A$ and $B$ is represented by $A \dot{+} B$, while

$$
A \stackrel{\vec{s}}{=} B
$$

means that $A$ is similar to $B$.

In this paper we shall find explicitly the generators of the group $\mathfrak{B}_{2 n}$ of all automorphisms of $\mathfrak{B}_{2 n}$, thereby obtaining a complete description of these automorphisms. This generalizes the result due to $\operatorname{Schreier}\left({ }^{1}\right)$ for the case $n=1$.

We shall frequently refer to results of an earlier paper: Automorphisms of the unimodular group, L. K. Hua and I. Reiner, Trans. Amer. Math. Soc. vol. 71 (1951) pp. 331-348. We designate this paper by AUT.

1. The commutator subgroup of $\mathfrak{B}_{2 n}$. The following useful result is an immediate consequence of the corresponding theorem for $\mathfrak{M}_{2 n}$ (AUT, Theorem 1).

TheOREM 1. Let $\mathfrak{S}_{2 n}$ be the commutator subgroup of $\mathfrak{P}_{2 n}$. Then clearly $\mathfrak{\Im}_{2 n} \subset \mathfrak{P}_{2 n}^{+}$. For $n=1, \mathfrak{S}_{2 n}$ is of index 2 in $\mathfrak{P}_{2 n}^{+}$, while for $n>1, \mathfrak{\Im}_{2 n}=\mathfrak{P}_{2 n}^{+}$.

THEOREM 2. In any automorphism of $\mathfrak{P}_{2 n}$, always $\mathfrak{P}_{2 n}^{+}$goes into itself.

Proof. This is a corollary to Theorem 1 when $n>1$, since the commutator subgroup goes into itself under any automorphism. For $n=1$, suppose that $\pm S \rightarrow \pm S_{1}$ and $\pm T \rightarrow \pm T_{1}$, where

$$
S=\left(\begin{array}{rr}
0 & 1 \\
-1 & 0
\end{array}\right), \quad T=\left(\begin{array}{ll}
1 & 1 \\
0 & 1
\end{array}\right)
$$

Since $S$ and $T$ generate $\mathfrak{M}_{2}^{+}$, it follows that $\pm S$ and $\pm T$ generate $\mathfrak{P}_{2}^{+}$,

Received by the editors May 18, 1951.

(1) Abh. Math. Sem. Hamburgischen Univ. vol. 3 (1924) p. 167. 
and hence so must $\pm S_{1}$ and $\pm T_{1}$. It is therefore sufficient to prove that $\operatorname{det} S_{1}=\operatorname{det} T_{1}=+1$. From $(S T)^{3}=I$ we deduce $S_{1} T_{1}= \pm T_{1}^{-1} S_{1}^{-1} T_{1}^{-1} S_{1}^{-1}$, so that det $S_{1} T_{1}=1$. Hence either $S_{1}$ and $T_{1}$ are both in $\mathfrak{P}_{2}^{+}$or both in $\mathfrak{P}_{2}^{-}$; we shall show that the latter alternative is impossible.

Suppose that $\operatorname{det} S_{1}=\operatorname{det} T_{1}=-1$. From $S^{2}=I$ we deduce $S_{1}^{2}= \pm I$; if $S_{1}^{2}=-I$, then $S_{1}^{2}+I=0$ and the characteristic equation of $S_{1}$ is $\lambda^{2}+1=0$, from which it follows that $\operatorname{det} S_{1}=1$; this contradicts our assumption that $\operatorname{det} S_{1}=-1$, so of necessity $S_{1}^{2}=I$. But if this is the case, then it is easy to show that there exists a matrix $A \in \mathfrak{M}_{2}$ such that $A S_{1} A^{-1}$ takes one of the two canonical forms

$$
\left(\begin{array}{rr}
1 & 0 \\
0 & -1
\end{array}\right) \text { and }\left(\begin{array}{rr}
1 & 0 \\
1 & -1
\end{array}\right) .
$$

By considering instead of the original automorphism $\tau$, a new automorphism $\tau^{\prime}$ defined by: $X^{\tau^{\prime}}=A X^{\tau} A^{-1}$, we may hereafter assume that

$$
S_{1}= \pm\left(\begin{array}{rr}
1 & 0 \\
0 & -1
\end{array}\right) \text { or } \pm\left(\begin{array}{rr}
1 & 0 \\
1 & -1
\end{array}\right)
$$

Let

$$
T_{1}= \pm\left(\begin{array}{ll}
a & b \\
c & d
\end{array}\right)
$$

then $a d-b c=-1$.

Now we observe that $J=(1) \dot{+}(-1)$ is distinct from $\pm I$ and $\pm S$, that it commutes with $S$, and that $J T$ is an involution. Hence there exists a matrix $M \in \mathfrak{P}_{2}$ distinct from $\pm I$ and $\pm S_{1}$, such that $M$ commutes with $S_{1}$, and $M T_{1}$ is an involution.

Case 1.

$$
S_{1}= \pm\left(\begin{array}{rr}
1 & 0 \\
0 & -1
\end{array}\right)
$$

Since $\left(S_{1} T_{1}\right)^{3}= \pm I$, we find that $a-d= \pm 1$. The only matrices commuting with $S_{1}$ which are distinct from $\pm I$ and $\pm S_{1}$ are

$$
\pm\left(\begin{array}{ll}
0 & 1 \\
1 & 0
\end{array}\right) \text { and } \pm\left(\begin{array}{rr}
0 & 1 \\
-1 & 0
\end{array}\right) \text {. }
$$

If $M$ is either of the first two matrices, then the condition that $M T_{1}$ be an involution yields $b+c=0$. Thus $a=d \pm 1, b=-c$, and $a d-b c=-1$. Combining these, we obtain $d(d \pm 1)+c^{2}=-1$, which is impossible. The other two choices for $M$ imply $b=c$, and therefore $d(d \pm 1)-c^{2}=-1$. Hence $1-4\left(1-c^{2}\right)$ is a perfect square; but $4 c^{2}-3=f^{2}$ implies $(2 c+f)(2 c-f)=1$, whence $c= \pm 1$. 
But then $a d=0$; from $a-d= \pm 1$ we deduce that $a^{2}-d^{2}= \pm 1$, whence $\left(S_{1} T_{1}^{2}\right)^{3}$ $= \pm I$, which is impossible.

Case 2.

$$
S_{1}= \pm\left(\begin{array}{rr}
1 & 0 \\
1 & -1
\end{array}\right) \text {. }
$$

From $\left(S_{1} T_{1}\right)^{3}= \pm I$ we obtain $a-d+b= \pm 1$. For $M$ there are the four possibilities

$$
\pm\left(\begin{array}{ll}
1 & -2 \\
0 & -1
\end{array}\right) \text { and } \pm\left(\begin{array}{ll}
1 & -2 \\
1 & -1
\end{array}\right)
$$

Since $M T_{1}$ is an involution, in the first two cases we have $a-2 c-d=0$, whence

$$
a d-b c=\left\{(a+d)^{2}+(a-d \pm 1)^{2}-1\right\} / 4 \neq-1 .
$$

In the second two cases we find that $a-2 c+b-d=0$, so that $2 c=a+b-d$ $= \pm 1$, which is again a contradiction. This completes the proof of Theorem 2 .

2. Automorphisms of $\mathfrak{P}_{2}^{+}$. Let us now determine all automorphisms of $\mathfrak{P}_{2}$. Since every such automorphism takes $\mathfrak{B}_{2}^{+}$into itself, we begin by considering all automorphisms of $\mathfrak{P}_{2}^{+}$.

THEOREM 3. Every automorphism of $\mathfrak{B}_{2}^{+}$is of the form $X \in \mathfrak{B}_{2}^{+} \rightarrow A X A^{-1}$ for some $A \in \mathfrak{M}_{2}$; that is, all automorphisms of $\mathfrak{B}_{2}^{+}$are "inner" (with $A \in \mathfrak{M}_{2}$ rather than $A \in \mathfrak{B}_{2}^{+}$.)

Proof. Let $\tau$ be any automorphism of $\mathfrak{P}_{2}^{+}$, and define $S$ and $T$ as before; let $S_{0} \in \mathfrak{M}_{2}$ be a fixed representative of $\pm S^{\top}$. By Theorem $2, S_{0} \in \mathfrak{M}_{2}^{+}$, and therefore $S_{0}^{2}=-I$. Let $T_{0}$ be that representative of $\pm T^{\tau}$ for which $\left(S_{0} T_{0}\right)^{3}=I$ is valid. Then $S \rightarrow S_{0}, T \rightarrow T_{0}$ induces a mapping from $\mathfrak{M}_{2}^{+}$onto itself. The mapping is one-to-one, for a'lthough an element of $\mathfrak{M}_{2}^{+}$can be expressed in many different ways as a product of powers of $S$ and $T$, these expressions can be gotten from one another by use of $S^{2}=-I,(S T)^{3}=I$; since $S_{0}$ and $T_{0}$ satisfy these same relations, the mapping is one-to-one. It is an automorphism because $\tau$ is one. Therefore (AUT, Theorem 2) there exists an $A \in \mathfrak{M}_{2}$ such that $S_{0}= \pm A S A^{-1}, T_{0}= \pm A T A^{-1}$. This proves the result.

Corollary. Every automorphism of $\mathfrak{B}_{2}$ is of the form $X \in \mathfrak{B}_{2} \rightarrow A X A^{-1}$ for some $A \in \mathfrak{M}_{2}$.

(This corollary is a simple consequence of Theorem 3, as is shown in AUT by the remarks following the statement of Theorem 4.)

3. The generators of $\mathfrak{B}_{2 n}$. Our main result may be stated as follows:

THEOREM 4. The generators of $\mathfrak{B}_{2 n}$ are

(i) The set of all inner automorphisms: 
and

(ii) The automorphism $\pm X \in \mathfrak{P}_{2 n} \rightarrow \pm X^{\prime-1}$.

REMARK. For $n=1$, the automorphism (ii) is a special case of (i).

In the proof of Theorem 4 by induction on $n$, the following lemma (which has already been established for $n=1$ ) will be basic:

LEMma 1. Let $J_{1}=(-1)+I^{(2 n-1)}$. In any automorphism $\tau$ of $\mathfrak{P}_{2 n}, J_{1}^{\tau}$ $= \pm A J_{1} A^{-1}$ for some $A \in M_{2 n}$.

Proof. The result is already known for $n=1$. Hereafter let $n \geqq 2$. Certainly $\left(J_{1}^{\tau}\right)^{2}= \pm I$ and det $J_{1}^{\tau}=-1$. If $\left(J_{1}^{\tau}\right)^{2}=-I$, then the minimum function of $J_{1}^{\tau}$ is $\lambda^{2}+1$, and its characteristic function must be some power of $\lambda^{2}+1$, whence $\operatorname{det} J_{1}^{\tau}=1$. Therefore $\left(J_{1}^{\tau}\right)^{2}=I$ is valid in $\mathfrak{M}_{2 n}$. After a suitable inner automorphism, we may assume that

where

$$
J_{1}^{\tau}=W(x, y, z)=L+\cdots+L \dot{+}(-I)^{(y)}+I^{(z)},
$$

$$
L=\left(\begin{array}{rr}
1 & 0 \\
1 & -1
\end{array}\right)
$$

occurs $x$ times, $2 x+y+z=2 n$, and $x+y$ is odd. (This follows from AUT, Lemma 1.)

Let $\mathfrak{S}_{1}$ be the group consisting of all elements of $\mathfrak{P}_{2 n}$ which commute with $J_{1}$, and $S_{2}$ the corresponding group for $J_{1}^{\tau}$. The lemma will be proved if we can show that $\mathfrak{S}_{1}$ is not isomorphic to $\mathfrak{H}_{2}$ unless $J_{1}^{r}= \pm J_{1}$. The group $\mathfrak{S}_{1}$ consists of the matrices $\pm\left(1+X_{1}\right) \in \mathfrak{P}_{2 n}$, so that $\mathfrak{S}_{1} \cong \mathfrak{M}_{2 n-1}$. The number of nonsimilar involutions in $\$_{1}$ is therefore $n(n+1)$ (see AUT, $\$ 4$ ). We shall prove that $\mathfrak{S}_{2}$ contains more than $n(n+1)$ involutions which are nonsimilar in $\mathfrak{H}_{2}$, except when $x=0, y=1, z=2 n-1$ or $x=0, y=2 n-1, z=1$.

Those elements $\pm C \in \mathfrak{P}_{2 n}$ which commute with $W$ must satisfy one of the two equations: $C W=W C$ or $C W=-W C$. The solutions of the first of these equations form a subgroup of $\mathrm{BS}_{2}$, and this subgroup is known (see AUT, proof of Lemma 2) to be isomorphic to $\mathfrak{S}_{0}=\mathfrak{S}_{0}(x, y, z)$ consisting of all matrices in $\mathfrak{B}_{2 n}$ of the form

$$
\left(\begin{array}{cc}
S_{1} & 2 R_{1} \\
Q_{1} & T_{1}
\end{array}\right)+\left(\begin{array}{cc}
S_{2} & Q_{2} \\
2 R_{2} & T_{2}
\end{array}\right)
$$

where $S_{1}, S_{2}, T_{1}$, and $T_{2}$ are square matrices of dimensions $x, x, z$, and $y$ respectively, and where $S_{1} \equiv S_{2}(\bmod 2), 2 x+y+z=2 n$, and $x+y$ and $x+z$ are both odd.

Next we prove that $\bar{C} W=-W \bar{C}$ is solvable only when $y=z$. The space 
$\mathfrak{U}$ of vectors $\mathfrak{u}$ such that $W \mathfrak{u}=\mathfrak{u}$ is of dimension $x+z$, while the space $\mathfrak{B}$ of vectors $\mathfrak{v}$ for which $W \mathfrak{b}=-\mathfrak{v}$ has dimension $x+y$. But if $\bar{C} W=-W \bar{C}$, then $W \bar{C} \mathfrak{u}=-\bar{C} \mathfrak{u}$ and $W \bar{C}^{-1} \mathfrak{v}=\bar{C}^{-1} \mathfrak{b}$, so the dimensions of $\mathfrak{U}$ and $\mathfrak{B}$ must be the same, whence $y=z$. Hence if $y \neq z$, there are no solutions of $\bar{C} W=-W \bar{C}$, $\bar{C} \in \mathfrak{M}_{2 n}$.

We may now proceed to find a lower bound for the number of nonsimilar matrices in $\mathfrak{S}_{0}(x, y, z)$. We briefly denote the elements of $B_{0}$ by $A \dot{+} B$, where

$$
A=\left(\begin{array}{cc}
S_{1} & 2 R_{1} \\
Q_{1} & T_{1}
\end{array}\right) \text { and } B=\left(\begin{array}{cc}
S_{2} & Q_{2} \\
2 R_{2} & T_{2}
\end{array}\right) .
$$

If $A_{1} \dot{+} B_{1}$ and $A_{2} \dot{+} B_{2}$ are two distinct involutions in $\mathbb{S}_{0}$, where either

$$
A_{1} \stackrel{s}{\neq} A_{2} \quad \text { in } \quad M_{x+z} \quad \text { or } \quad B_{1} \not{s} B_{2} \text { in } M_{x+y}
$$

then certainly

$$
A_{1}+B_{1} \stackrel{s}{\neq} A_{2}+B_{2} \text { in } \mathfrak{s}_{0}
$$

Now let

$$
\begin{aligned}
& A=I^{\left(a_{1}\right)}+(-I)^{\left(b_{1}\right)}+L+\cdots+L, \\
& B=I^{\left(a_{2}\right)}+(-I)^{\left(b_{2}\right)}+L+\cdots+L,
\end{aligned}
$$

where $L$ occurs $c_{1}$ times in $A$ and $c_{2}$ times in $B$; the various elements $A+B$ gotten by taking different sets of values of $\left(a_{1}, b_{1}, c_{1}, a_{2}, b_{2}, c_{2}\right)$, if they lie in $\mathcal{B}_{0}$, are certainly nonsimilar in $\mathcal{B}_{0}$, except that $A+B$ and $(-A) \dot{+}(-B)$ are the same element of $\mathfrak{S}_{0}$. Hence the number $N$ of nonsimilar involutions of $\mathbb{G}_{0}$ is at least half of the number $N_{1}$ of solutions of

$$
\begin{aligned}
& a_{1}+b_{1}+2 c_{1}=x+z, \\
& a_{2}+b_{2}+2 c_{2}=x+y,
\end{aligned}
$$

where if $x \neq 0$ we impose the restrictions that $c_{1} \leqq(z+1) / 2, c_{2} \leqq(y+1) / 2$, and that in $B$ instead of $L$ we use $L^{\prime}$. (These conditions insure that $A+B \in\left(\mathcal{B}_{0}\right.$.) As in the previous paper, one readily shows that $N>n(n+1)$ unless $J_{1}^{r}= \pm J_{1}$. We omit the details.

This leaves only the case where $y=z$. If $\bar{C} W=-W \bar{C}$, then $\bar{C}^{k} W$ $=(-1)^{k} W \bar{C}^{k}$; therefore no odd power of $\bar{C}$ can be $\pm I$. Let $p$ be a prime such that $n<p<2 n$. Since $x+y=n$, certainly $n$ is odd, and $p \geqq n+2$. Now $\mathfrak{G}_{1}$ (being isomorphic to $\mathfrak{M}_{2 n-1}$ ) contains infinitely many elements of order $p$. However, $\mathrm{G}_{2}$ contains only two such elements, since $\bar{C}^{p} \neq \pm I$ by the above argument, while if $C \in \mathbb{S}_{0}$ and $C^{p}= \pm I$, then setting $C=A^{(n)} \dot{+} B^{(n)}$ shows that $A^{p}= \pm I$ and $B^{p}= \pm I$. However, $A \in \mathfrak{M}_{n}$, and if $A^{p}= \pm I$, then the minimum function of $A$ must divide $\lambda^{p} \mp 1$. But the degree of the minimum function is at most $n$, and therefore is less than $p-1$, whereas $\lambda^{p} \mp 1$ is the 
product of a linear factor $\lambda \mp 1$ and an irreducible factor of degree $p-1$; thence the minimum function of $A$ is $\lambda \mp 1$, so $A= \pm I$. In the same way $B= \pm I$. Hence the only solutions are $C=I^{(n)} \dot{+} I^{(n)}$ and $C=-I^{(n)} \dot{+} I^{(n)}$. This completes the proof of the lemma. We remark that the use of the existence of the prime $p$ could have been avoided, but the proof is much quicker this way.

4. Proof of the main theorem. We are now ready to prove Theorem 4 by induction on $n$. Hereafter, let $n \geqq 2$ and assume that Theorem 4 holds for $n-1$. Let $\tau$ be any automorphism of $\mathfrak{B}_{2 n}$; then by Lemma $1, J_{1}^{\tau}= \pm A J_{1} A^{-1}$ for some $A \in \mathfrak{M}_{2 n}$. If we change $\tau$ by a suitable inner automorphism, we may assume that $J_{1}^{\tau}= \pm J_{1}$.

Therefore, every $M \in \mathfrak{P}_{2 n}$ which commutes with $J_{1}$ goes into another such element, that is,

$$
\pm\left[\begin{array}{ll}
1 & \mathfrak{n}^{\prime} \\
\mathfrak{n} & X
\end{array}\right]^{\tau}= \pm\left[\begin{array}{ll}
1 & \mathfrak{n}^{\prime} \\
\mathfrak{n} & Y
\end{array}\right]
$$

where $\mathfrak{n}$ denotes a column vector all of whose components are zero, and $X \in \mathfrak{M}_{2 n-1}$. Thus, $\tau$ induces an automorphism on $\mathfrak{M}_{2 n-1}$. Consequently (AUT, Theorem 4) there exists a matrix $A \in \mathfrak{M}_{2 n-1}$ such that $Y=A X^{*} A^{-1}$ for all $X \in \mathfrak{M}_{2 n-1}$, where either $X^{*}=X$ for all $X \in \mathfrak{M}_{2 n-1}$ or $X^{*}=X^{\prime-1}$ for all $X$ $\in \mathfrak{M}_{2 n-1}$. After a further inner automorphism by a factor of $(1) \dot{+} A^{-1}$, we may assume that $J_{1}^{\tau}= \pm J_{1}$ and also that $X^{\tau}=Y=X^{*}$ for all $X \in \mathfrak{M}_{2 n-1}$. Then

Let $J_{\nu}$ be obtained from $I^{(2 n)}$ by replacing the $\nu$ th diagonal element by -1 .

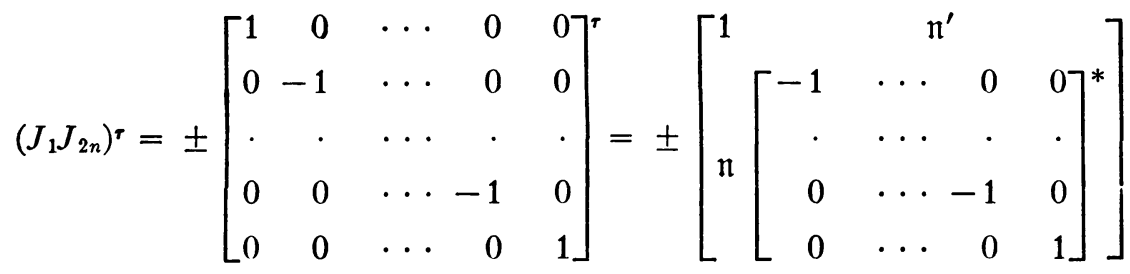

$$
\begin{aligned}
& = \pm J_{1} J_{2 n},
\end{aligned}
$$

so that $\pm J_{2 n}$ is invariant. Similarly, all of the matrices $\pm J_{\nu}(\nu=1, \cdots, 2 n)$ are invariant. Therefore for any $X \in \mathfrak{M}_{2 n-1}$ we have

$$
\pm\left(\begin{array}{ll}
1 & \mathfrak{n}^{\prime} \\
\mathfrak{n} & X
\end{array}\right)^{\tau}= \pm\left(\begin{array}{cc}
1 & \mathfrak{n}^{\prime} \\
\mathfrak{n} & A_{1} X^{*} A_{1}^{-1}
\end{array}\right), \cdots, \pm\left(\begin{array}{cc}
X & \mathfrak{n} \\
\mathfrak{n}^{\prime} & 1
\end{array}\right)^{\tau}= \pm\left(\begin{array}{cc}
A_{2 n} X^{*} A_{2 n}^{-1} & \mathfrak{n} \\
\mathfrak{n}^{\prime} & 1
\end{array}\right)
$$

with $A_{\nu} \in \mathfrak{M}_{2 n-1}$, and in fact $A_{1}=I$.

Now suppose that $Z \in \mathfrak{M}_{2 n-2}$, and consider $\pm\left(Z \dot{+} I^{(2)}\right)$; since it commutes with $J_{2 n-1}$ and $J_{2 n}$, so does its image. But therefore 


$$
A_{2 n}\left(\begin{array}{ll}
Z & \mathfrak{n} \\
\mathfrak{n}^{\prime} & 1
\end{array}\right) A_{2 \mathfrak{n}}^{-1}=\left(\begin{array}{ll}
\bar{Z} & \mathfrak{n} \\
\mathfrak{n}^{\prime} & 1
\end{array}\right)
$$

where $\bar{Z}$ denotes some matrix in $\mathfrak{M}_{2 n-2}$. From this one easily deduces that $A_{2 n}$ must be of the form $B \dot{+}(1)$, with $B \in \mathfrak{M}_{2 n-2}$. By considering the matrices commuting with $J_{\nu}$ and $J_{2 n}$ for $\nu=1, \cdots, 2 n-2$ we see that $A_{2 n}$ must be diagonal. Furthermore, it is clear that all of the $A_{v}(\nu=1, \cdots, 2 n)$ must be diagonal, and all are sections of one diagonal matrix $D^{(2 n)}$. Using the further inner automorphism factor $D^{-1}$, we find that $\pm X^{r}= \pm X^{*}$ for every decomposable matrix $\pm X \in \mathfrak{P}_{2 n}$. Since $\mathfrak{P}_{2 n}$ is generated by the set of its decomposable matrices, the theorem is proved.

Tsing Hua University, Peking, China.

UNIVERSITY OF ILLINOIS, URBANA, IlL. 